\title{
Expression of human full-length MUC1 inhibits the proliferation and migration of a B16 mouse melanoma cell line
}

\author{
FENGLI WANG $^{1 *}$, QIONGSHU LI $^{1 *}$, WEIHUA NI $^{1}$, FANG FANG $^{1,2}$, XIAXIA SUN $^{1}$, \\ FEI XIE ${ }^{1}$, JUAN WANG $^{1}$, FANG WANG $^{1}$, SUJUN GAO $^{3}$ and GUIXIANG TAI ${ }^{1}$ \\ ${ }^{1}$ Department of Immunology, Norman Bethune College of Medicine, Jilin University, Changchun 130021; \\ ${ }^{2}$ Department of Immunology, Jilin Medical College, Jilin 132013; ${ }^{3}$ Department of Hematology and Oncology, \\ The First Bethune Hospital of Jilin University, Changchun 130021, P.R. China
}

Received January 7, 2013; Accepted March 26, 2013

DOI: $10.3892 / o r .2013 .2440$

\begin{abstract}
Mucin 1 (MUC1) is a large transmembrane glycoprotein that is aberrantly overexpressed in most adenocarcinomas and certain hematological malignancies. MUC1 is known to function as an oncogene with roles in both tumor formation and progression, making it a potential target for immunotherapy. B16-MUC1 cells with human full-length MUC1 are frequently used to study the antitumor activities of MUC1-based vaccines. However, we found that the growth of B16-MUC1 cells was significantly reduced in vitro. Therefore, in this study, we established two MUC1-positive clones, B16-MUC1 9-12 and B16-MUC1 9-23, and one empty vector control clone, B16-neo, to investigate the effects of MUC1 on the cancer-related characteristics of B16 cells in vitro and in vivo. Our results demonstrated that, compared with MUC1negative cells, cells expressing MUC1 exhibited decreased cell proliferation, increased cell cycle arrest and reduced cell migratory and invasive capacities. We further investigated several MUC1-related molecules of the $\beta$-catenin pathway, and found that the expression of MUC1 decreased the translocation of $\beta$-catenin into the nucleus, reduced the activity of $\mathrm{T}$ cell factor (TCF) and blocked the expression of cyclin D1 and c-Myc. Moreover, when inoculated into BALB/c nude mice, cells expressing MUC1 developed smaller tumors compared with the control cells. These results demonstrate that MUC1 expression negatively affects the malignancy of B16 cells, and
\end{abstract}

Correspondence to: Professor Sujun Gao, Department of Hematology and Oncology, The First Bethune Hospital of Jilin University, 71 Xinmin Street, Changchun 130021, P.R. China E-mail: drsujung@gmail.yahoo.com.cn

Professor Guixiang Tai, Department of Immunology, Norman Bethune College of Medicine, Jilin University, 126 Xinmin Street, Jilin, Changchun 130021, P.R. China

E-mail: taiguixiang@163.com

*Contributed equally

Key words: mucin 1, B16, proliferation, migration, growth inhibition suggest that the regulatory mechanisms of MUC1 as an oncoprotein are more complex than previously appreciated.

\section{Introduction}

Mucin 1 (MUC1) is a transmembrane glycoprotein that is expressed in most epithelial cells and is aberrantly overexpressed in many types of human adenocarcinomas and hematologic malignancies (1). MUC1 consists of a large extracellular $\mathrm{N}$-terminal subunit and a $\mathrm{C}$-terminal subunit that resides on the cell surface as a heterodimeric complex via strong noncovalent interactions. The $\mathrm{N}$-terminal subunit consists of a variable number of 20-amino acid tandem repeats (VNTR) that comprise the majority of the extracellular domain. The C-terminal subunit is composed of a 58-amino acid extracellular domain, a 28-amino acid transmembrane domain (TM) and a 72-amino acid cytoplasmic tail (CT) (2-4). The MUC1-CT is highly conserved among different species and possesses 7 tyrosine residues that can be potentially phosphorylated by multiple kinases (5-8); this region also associates with certain transcription factors (9-11). Available evidence indicates that the MUC1-CT is involved in many signaling pathways, including the Wnt/ $\beta$-catenin $(5,12,13)$, p53 $(9,11)$ and NF- $\kappa \mathrm{B}$ (14) pathways. $\beta$-catenin, a major effector of the Wnt signaling pathway, interacts with the MUC1-CT at an SXXXXXSSLS site. This interaction blocks GSK3 $\beta$-induced degradation of $\beta$-catenin and promotes the translocation of $\beta$-catenin to the nucleus, where it forms complexes with the LEF/TCF (lymphoid enhancer factor/T cell factor) transcription factors and activates transcription of Wnt-responsive genes such as cyclin D1 and c-Myc to regulate cell proliferation $(15,16)$.

Previous studies have shown that MUC1 plays a role in a diverse array of cellular processes including differentiation (17) motility or inhibition of cell-cell and cell-matrix adhesion (18-21) and immune regulation (22). Recently, the majority of studies have shown that the MUC1-CT contributes to malignant transformation as an oncoprotein. Overexpression of the MUC1-CT in 3Y1 fibroblasts induced cellular transformation and promoted tumor formation in nude mice (23). Mutation of the MUC1-CT (Y46F, Y60F) abrogated MUC1-induced anchorage-independent growth and tumorigenicity in human 
Table I. Primer sequences and reaction parameters used for PCR analysis.

\begin{tabular}{|c|c|c|c|c|}
\hline Gene name & $\begin{array}{c}\text { Primers } \\
\left(5^{\prime}-3^{\prime}, \text { forward and reverse }\right)\end{array}$ & $\begin{array}{c}\text { Annealing } \\
\text { temperature }\left({ }^{\circ} \mathrm{C}\right)\end{array}$ & Cycles & $\begin{array}{l}\text { Product } \\
\text { size (bp) }\end{array}$ \\
\hline \multirow[t]{2}{*}{ Human MUC1 } & Forward: 5'-TGAGTGATGTGCCATTTCC-3' & & & \\
\hline & Reverse: 5'-CTGCCCGTAGTTCTTTCG-3' & 56 & 30 & 158 \\
\hline \multirow[t]{2}{*}{ Mouse $\beta$-actin } & Forward: 5'-TGTCCACCTTCCAGCAGATGT-3' & & & \\
\hline & Reverse: 5'-AGCTCAGTAACAGTCCGCCTAG-3' & 54 & 25 & 101 \\
\hline \multirow[t]{2}{*}{ Mouse cyclin D1 } & Forward: 5'-AGCAGAAGTGCGAAGAGG-3' & & & \\
\hline & Reverse: 5'-GCAGTCAAGGGAATGGTC-3' & 52 & 30 & 154 \\
\hline \multirow[t]{2}{*}{ Mouse c-Myc } & Forward: 5'-AAGGGAAGACGATGACGG-3' & & & \\
\hline & Reverse: 5'-TGAGAAACCGCTCCACATA-3' & 52 & 40 & 172 \\
\hline
\end{tabular}

PCR, polymerase chain reaction.

colon carcinoma cells $(24,25)$. Inhibition of the MUC1-CT induced cancer cell death and tumor regression $(26,27)$. The deletion of MUC1 expression from MMTV-Wnt-1 transgenic mice resulted in a significant increase in the time to mammary gland tumor onset (28). However, several studies have shown an inverse association between MUC1 and cell proliferation and adhesion. For example, Hattrup and Gendler (29) and Costa et al (30) demonstrated that MUC1 downregulation in human BT20 breast carcinoma cells or human gastric carcinoma MKN45 cells increased proliferation. Considering these contradictory findings, the role of MUC1 in cancer progression has yet to be clarified.

In this study, we investigated the effects and related mechanisms of MUC1 on cancer-related characteristics of B16 cells by stable expression of the human full-length MUC1 in B16 cells. We found that MUC1 expression in B16 cells inhibited cell proliferation, decreased cell migration and invasion, and suppressed tumor growth in BALB/c nude mice. These results suggest that the modulatory effects of MUC1 in tumor cells may be more complex than previously appreciated, which reinforces the importance of understanding alternative regulatory mechanisms of MUC1.

\section{Materials and methods}

Cell line, plasmids and animals. The B16 cell line was purchased from ATCC (Manassas, VA, USA) and cultured in Iscove's modified Dulbecco's medium (IMDM) supplemented with $100 \mathrm{U} / \mathrm{ml}$ penicillin, $100 \mu \mathrm{g} / \mathrm{ml}$ streptomycin, and $10 \%$ fetal bovine serum (FBS) (Gibco-BRL, Carlsbad, $\mathrm{CA}, \mathrm{USA})$ in a humidified atmosphere of $5 \% \mathrm{CO}_{2}$ at $37^{\circ} \mathrm{C}$. The pcDNA3-MUC1 plasmid, which contains the full-length human MUC1 consisting of 22 TR, was a gift from Dr O.J. Finn of the University of Pittsburgh (Pittsburgh, PA, USA). BALB/c nude mice (4-6 weeks old) were purchased from Vital River Laboratories (Vital River, China). Animals were maintained in specific pathogen-free conditions and were fed sterile water and food ad libitum. All animals were treated in accordance with the National Institutes of Health Guide for the Care and Use of Laboratory Animals and with the approval of the
Scientific Investigation Board of Science and Technology of Jilin Province.

Cell transfection. B16 cells growing in an exponential phase were seeded in a 6-well plate. When cells reached $80-90 \%$ confluence, $1.0 \mu \mathrm{g}$ of pcDNA3-MUC1 plasmid was transfected with Lipofectamine ${ }^{\mathrm{TM}} 2000$ (Invitrogen Life Technologies, Carlsbad, CA, USA) according to the manufacturer's protocol. Two stable MUC1-positive clones (B16-MUC1 9-12, B16-MUC1 9-23) were selected in 1,000 $\mu \mathrm{g} / \mathrm{ml} \mathrm{G} 418$ (Gibco-BRL), and the concentration was decreased to $600 \mu \mathrm{g} /$ $\mathrm{ml}$ to maintain filtrate efficacy. Meanwhile, a negative control B16-neo cell line was prepared by transfecting the pcDNA3 empty vector into the B16 cells.

Reverse transcription-polymerase chain reaction (RT-PCR). MUC1, cyclin D1 and c-Myc mRNA levels were analyzed by RT-PCR. Total RNA was extracted from cells using Trizol reagent (Invitrogen Life Technologies). Total RNA was converted to cDNA using M-MLV reverse transcriptase and Oligo(dT) primers (Promega Corporation, Madison, WI, USA) according to the manufacturer's protocol. The reverse transcribed products were used to amplify MUC1, cyclin D1 and c-Myc by PCR using Ex-Taq DNA polymerase (Takara Bio, Inc., Shiga, Japan), and $\beta$-actin was used as an internal control gene. The primer sequences and reaction parameters are shown in Table I. Amplified products were analyzed on a $1.5 \%$ agarose gel, and DNA was visualized by a Gel Image System (Tanon). The final value was expressed as a ratio of the relative density of the target gene to $\beta$-actin from three independent experiments (means $\pm \mathrm{SD}$ ).

Flow cytometry. To analyze MUC1 expression of stable transfectants, cells $\left(1 \times 10^{6}\right)$ were fixed with paraformaldehyde for $1 \mathrm{~h}$ and washed twice with fluorescence-activated cell sorter (FACS) solution (PBS containing 2\% FCS and $0.1 \% \mathrm{NaN}_{3}$ ). Subsequently, the cells were incubated with a mouse monoclonal antibody against MUC1 tandem repeats (HMPV; BD Biosciences, Franklin Lakes, NJ, USA) on ice for $30 \mathrm{~min}$, washed twice with FACS solution and stained with fluorescein 
isothiocyanate (FITC)-conjugated goat anti-mouse secondary antibody (Proteintech Group, Chicago, IL, USA) at a dilution of 1:100 for $30 \mathrm{~min}$ on ice in the dark. After washing twice with FACS solution, the expression of MUC1 was analyzed by flow cytometry (FACSCalibur; BD Biosciences).

Immunofluorescence. Cells were fixed with $4 \%$ paraformaldehyde and permeabilized with $0.2 \%$ Triton X-100. After blocking with $2 \%$ bovine serum albumin (BSA), the cells were incubated with a mouse anti-MUC1 monoclonal antibody (GP1.4, NeoMarkers) overnight at $4^{\circ} \mathrm{C}$. After washing, PE-conjugated goat anti-mouse $\operatorname{IgG}$ (Proteintech Group) was added for $1 \mathrm{~h}$ at $37^{\circ} \mathrm{C}$ in the dark. The nuclei were stained with $1 \mu \mathrm{g} / \mathrm{ml}$ 4',6-diamidino-2-phenylindole (DAPI) (SigmaAldrich, St. Louis, MO, USA) for $10 \mathrm{~min}$, and the cells were visualized using an inverted fluorescence microscope (Olympus, IX71). Tumors were fixed in 10\% neutral-buffered formalin and embedded in paraffin. Serial paraffin sections $(5-\mu \mathrm{m})$ were cut and mounted on slides for immunofluorescence staining. Sections were treated with $1.5 \%$ rabbit serum at $37^{\circ} \mathrm{C}$ for $30 \mathrm{~min}$. Following that, the sections were incubated with primary antibody GP1.4 and PE-conjugated goat anti-mouse $\operatorname{IgG}$ as described above. The nuclei were stained with DAPI and the sections were visualized by inverted fluorescence microscopy.

Cell proliferation assay. Cell viability was determined using a WST-1 cell proliferation assay according to the manufacturer's protocol (Roche Diagnostics, Mannheim, Germany). Briefly, cells $\left(5 \times 10^{3} /\right.$ well $)$ were seeded in triplicate in 96-well plates and cultured at $37^{\circ} \mathrm{C}$ with $5 \% \mathrm{CO}_{2}$ in a humidified atmosphere for $96 \mathrm{~h}$. WST-1 reagent was added at $24,48,72$ or $96 \mathrm{~h}$, and incubation was continued for an additional 1-2 $\mathrm{h}$. Then, the absorbance was measured using a microplate reader at a wavelength of $450 \mathrm{~nm}$ (BioTek Instruments, Inc., Winooski, VT, USA). The resulting values were calculated as a ratio of B16-MUC1 to B16-neo and were the average from three independent experiments (means $\pm \mathrm{SD}$ ).

Cell cycle analysis. Cells $\left(1 \times 10^{6}\right)$ were harvested and then permeabilized with $70 \%$ ice-cold ethanol on ice for $30 \mathrm{~min}$. Cells were then washed and incubated in staining buffer with $50 \mu \mathrm{g} / \mathrm{ml}$ propidium iodide (PI), $10 \mu \mathrm{g} / \mathrm{ml} \mathrm{RNase} \mathrm{A} \mathrm{and} 0.1 \%$ Triton X-100 for $30 \mathrm{~min}$ in the dark. Subsequently, the cell cycle was analyzed by flow cytometry (FACSCalibur; BD Biosciences).

Cell migration and invasion assay. Cell migration and Matrigel invasion assays were performed using Transwell chambers with $8-\mu \mathrm{m}$ pore size filters (Corning Incorporated, Corning, NY, USA) coated with or without Matrigel matrix (BD Biosciences) in a 24-well plate. In each well, $6 \times 10^{4}$ cells were seeded to the upper chamber in $200 \mu \mathrm{l}$ IMDM containing $1 \%$ FBS, and $600 \mu 1$ IMDM containing 10\% FBS was added to the bottom chamber as a chemoattractant. The cells were incubated at $37^{\circ} \mathrm{C}$ in a $5 \% \mathrm{CO}_{2}$ atmosphere and allowed to migrate or invade for $36 \mathrm{~h}$. Following the incubation period, the remaining cells in the upper chamber were removed gently with a cotton swab. The cells on the lower surface of the chamber were fixed with methanol for $20 \mathrm{~min}$, and then stained with $1 \%$ crystal violet in $20 \%$ methanol for $30 \mathrm{~min}$. The cells that had migrated or invaded through the filters were counted in five random fields under a microscope.

Coimmunoprecipitation analysis. B16-MUC1 cells were lysed with RIPA lysis buffer containing $50 \mathrm{mM}$ Tris, $150 \mathrm{mM} \mathrm{NaCl}$, $1 \%$ Triton X-100, $1 \%$ sodium deoxycholate, $0.1 \%$ sodium dodecyl sulphate (SDS), $0.1 \mathrm{mM}$ ethylenediaminetetraacetic acid (EDTA), $1 \mathrm{mM}$ phenylmethyl sulfonyl fluoride (PMSF), $0.23 \mathrm{U} / \mathrm{ml}$ aprotinin, and $10 \mu \mathrm{M}$ leupeptin (Sigma-Aldrich). Protein concentrations were measured using a BCA protein assay kit (Beyotime Biotechnology, Jiangsu, China). Equal protein aliquots were subjected to immunoprecipitation with $1.0 \mu \mathrm{g}$ of mouse IgG or anti-MUC1-CT antibody (Ab-5; Neomarker) for $16 \mathrm{~h}$ at $4^{\circ} \mathrm{C}$ followed by precipitation with Protein $\mathrm{G}$ agarose beads (Promega Corporation). Immunoprecipitated proteins and total cell lysates were resolved by $10 \%$ sodium dodecyl sulphate-polyacrylamide gel electrophoresis (SDS-PAGE) and subjected to immunoblot analysis with anti- $\beta$-catenin (1:1000; BD Biosciences) for $16 \mathrm{~h}$ at $4^{\circ} \mathrm{C}$. Following incubation, the reactivity was detected with horseradish peroxidase-conjugated secondary antibodies (1:2000; Sigma-Aldrich) and ECL reagents (GE Healthcare).

Luciferase reporter assay. Cells were seeded in 6-well plates. When cells reached $90 \%$ confluence, $1.0 \mu \mathrm{g}$ of TOPflash and FOPflash plasmids (Upstate Biotechnology, Inc., Lake Placid, NY, USA) were transiently transfected with Lipofectamine ${ }^{\mathrm{TM}}$ according to the manufacturer's instructions. To normalize the transfection efficiency, the cells were cotransfected with $0.05 \mu \mathrm{g}$ of pRL-TK (Promega Corporation). Forty eight hours post-transfection, the luciferase assay was performed with the Dual Luciferase Assay System kit (Promega Corporation). Relative luciferase activity was calculated as the fold induction after normalization for transfection efficiency.

Western blot analysis. Cells were lysed with RIPA lysis buffer as described above. Nuclear and cytoplasmic protein extracts were isolated using a cytoplasmic and nuclear protein extraction kit (Thermo Scientific) according to the manufacturer's protocol. Protein concentrations were measured using a BCA protein assay kit (Beyotime Biotechnology). Equal amounts of protein were separated by $10 \%$ SDS-PAGE and transferred to PVDF membranes (Millipore, Billerica, MA, USA), and the membranes were blocked in $3 \%$ BSA overnight at $4^{\circ} \mathrm{C}$. The membranes were then incubated with primary antibodies against MUC1 (GP1.4) (1:2000), c-Myc (1:1000), cyclin D1 (1:1000; both from Epitomics, Burlingame, CA, USA), $\beta$-catenin (1:1000; BD Transdution Labs) or E-cadherin (1:800, Proteintech) for $2 \mathrm{~h}$ at room temperature, with the antibodies against $\beta$-actin (1:2000), IкB $\alpha$ (1:2000) and Lamin B1 (1:2000, all from Epitomics) as loading controls. Then, the membranes were incubated with horseradish peroxidase-conjugated secondary antibodies (1:2000; Sigma-Aldrich) for another $2 \mathrm{~h}$ at room temperature. The membranes were developed using ECL reagents (GE Healthcare). Each experiment was repeated at least 3 times.

In vivo tumor growth assays. To determine the effects of MUC1 expression on tumorigenesis in vivo, BALB/c female 


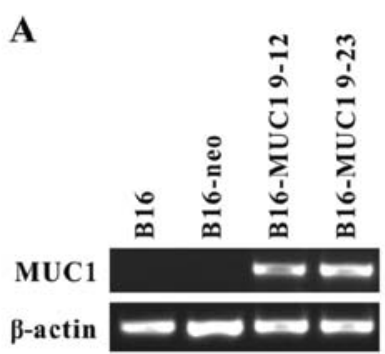

C
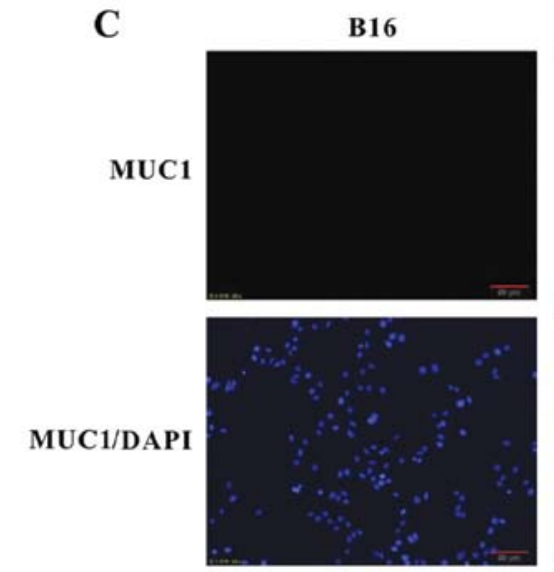

\section{B}
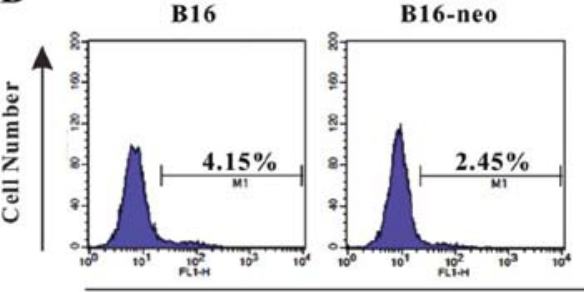

Intensity (MUC1-FITC)
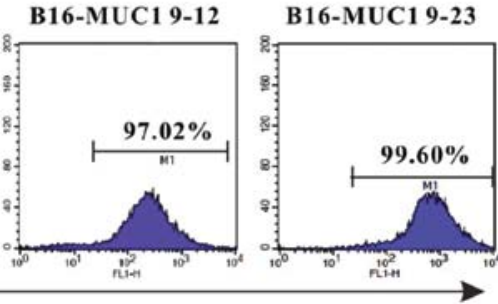

B16-MUC1 9-12
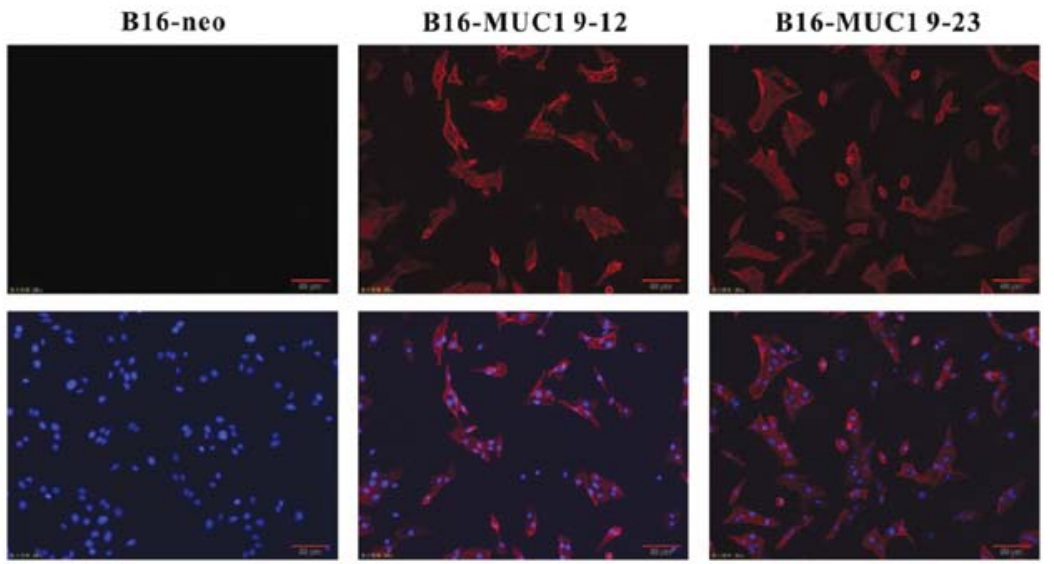

Figure 1. Stable mucin 1 (MUC1) expression was detected in transfected cells. (A) MUC1 mRNA expression was detected by reverse transcription-polymerase chain reaction (RT-PCR) with $\beta$-actin as an internal control reference gene. (B) Cells $\left(1 \times 10^{6}\right)$ were analyzed by flow cytometry for MUC1 expression using the anti-MUC1 primary antibody (HMPV) and FITC-conjugated secondary antibody. (C) MUC1 expression was detected by immunofluorescence staining with the anti-MUC1 primary antibody (GP1.4) and PE-conjugated secondary antibody (red). Nuclei were stained with DAPI (blue).

nude mice (4-6 weeks old) were used to establish a subcutaneous transplant tumor model. Mice were randomly divided into 2 groups ( 5 animals/group) that were designated as the B16-neo group and the B16-MUC1 group. B16-MUC1 cells or B16-neo cells $\left(2 \times 10^{6}\right)$ were subcutaneously injected into the right flank of each mouse. Tumor size was measured by calipers every 2 days. On day 12 post injection, the tumors were removed and weighed.

Statistical analysis. All statistical analyses were performed using unpaired Student's t-tests, and $\mathrm{P}<0.05$ was considered to indicate a statistically significant result.

\section{Results}

Stable MUC1 expression in mouse melanoma B16 cells. To assess the effects of MUC1 expression, mouse B16 cells were transfected with a vector encoding human full-length MUC1 containing 22 TR (pcDNA3-MUC1) or an empty pcDNA3 vector. Stable transfectants were selected with G418 $(1,000 \mu \mathrm{g} / \mathrm{ml})$ and analyzed for MUC1 expression by RT-PCR, flow cytometry and immunofluorescence. Two MUC1-positive clones (B16-MUC1 9-12 and B16-MUC1 9-23) expressed higher MUC1 mRNA levels and were selected for further study. By contrast, there was no detectable MUC1 expression in the B16-neo cells transfected with the empty vector (Fig. 1A). Flow cytometry (Fig. 1B) and immunofluorescence staining (Fig. 1C) with anti-MUC1 tandem repeat peptide antibodies (HMPV and GP1.4) verified that MUC1 was expressed on the cell surface of 97.0 and $99.0 \%$ of B16-MUC1 9-12 and B16-MUC1 9-23 cells, respectively.

MUC1 expression inhibits cell proliferation and induces G1-phase arrest in vitro. To determine the effect of MUC1 expression on cell growth in vitro, equal numbers of B16-MUC1, B16-neo and B16 cells were seeded in 96-well plates and cultured for $96 \mathrm{~h}$ in a humidified atmosphere of $5 \% \mathrm{CO}_{2}$ at $37^{\circ} \mathrm{C}$. Cell viability was evaluated by the WST-1 assay. The viability of B16-MUC1 cells was significantly reduced in a time-dependent manner when compared with the viability of the B16-neo or B16 cells $(\mathrm{P}<0.01)$ (Fig. 2A). We further analyzed the cell cycle of B16-MUC1, B16-neo and B16 cells by flow cytometry. The results showed that B16-MUC1 cells had a higher percentage of cells in the G0/G1 phase $(73.22 \pm 2.13 \%)$ and fewer in the $\mathrm{G} 2 / \mathrm{M}$ phase $(2.32 \pm 0.39 \%)$ when compared with B16-neo cells $(61.2 \pm 3.34 \%$ in $\mathrm{G} 0 / \mathrm{G} 1)$ $(6.07 \pm 1.26 \%$ in $\mathrm{G} 2 / \mathrm{M})$ or B16 cells $(57.68 \pm 3.41 \%$ in $\mathrm{G} 0 / \mathrm{G} 1)$ $(6.39 \pm 1.7 \%$ in G2/M) (Fig. 2B and C). These results indicate that MUC1 expression in B16 cells inhibited cell proliferation and induced cell cycle arrest at the G1 phase.

MUC1 expression inhibits cell migration and invasion in vitro. To investigate whether MUC1 expression affects the motility of B16 cells, the migratory capacity of cells was evaluated using the Transwell migration assay. The results showed that the number of B16-MUC1 cells that migrated into the lower chamber was significantly decreased when compared to the number of migrating B16-neo or B16 cells (Fig. 3A and B) 
A
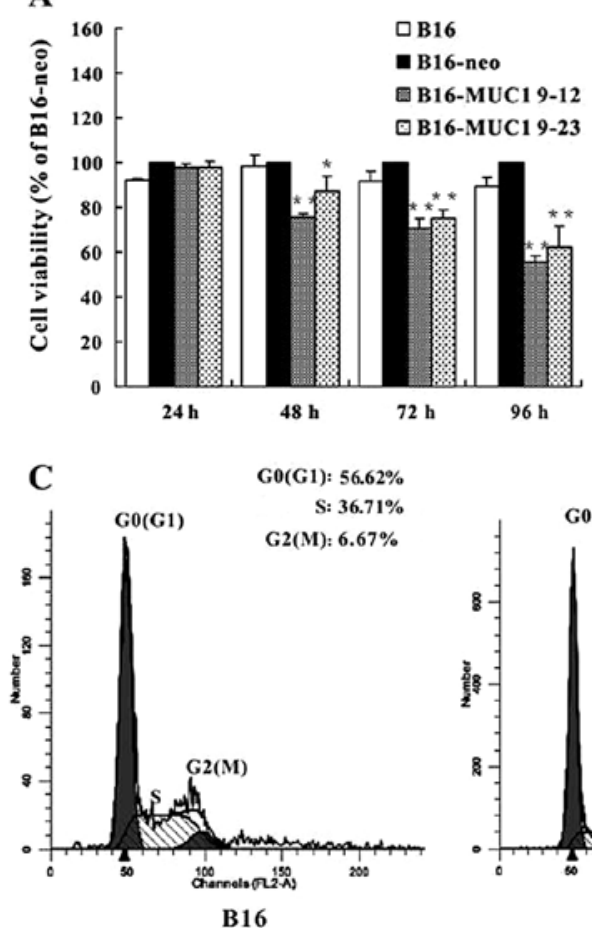

B

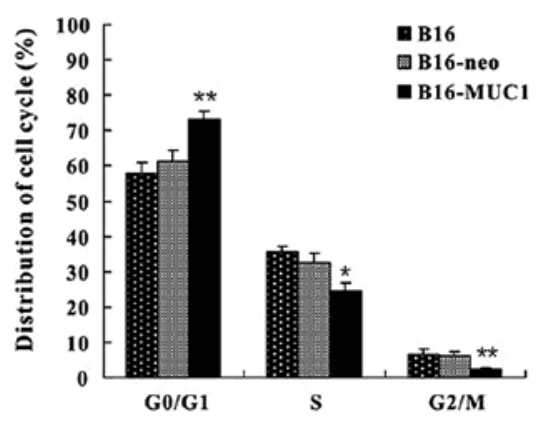

G0(G1): $64.98 \%$ S: $30.28 \%$

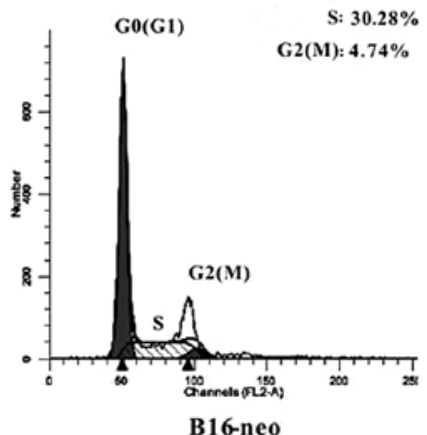

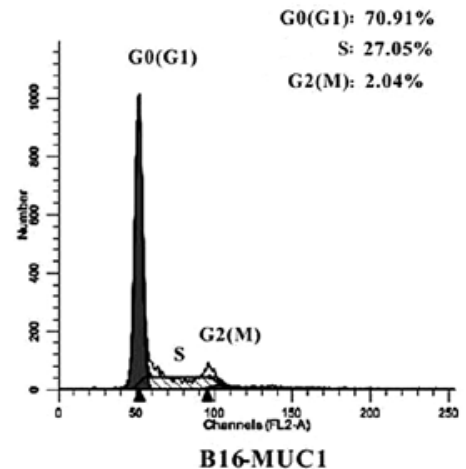

Figure 2. Mucin 1 (MUC1) expression inhibits cell proliferation and blocks cell cycle progression in transfected cells. (A) Cell proliferation was determined by the WST-1 assay. Triplicate wells containing 5x103 cells of B16, B16-neo or two stable MUC1-positive clones (B16-MUC1 9-12 and B16-MUC1 9-23) were evaluated for proliferation at $24 \mathrm{~h}$ intervals. Values were calculated from the relative optical density ratio of B16-MUC1/B16-neo and B16/B16-neo cells (B and C) Cells $\left(1 \times 10^{6}\right)$ were collected, permeabilized and stained with $50 \mu \mathrm{g} / \mathrm{ml}$ propidium iodide (PI) in the presence of RNase A. The distribution of cells in the G0/G1, S and G2/M phases was analyzed by flow cytometry. Data are expressed as the means $\pm \mathrm{SD}$ of 3 independent experiments. ${ }^{*} \mathrm{P}<0.05,{ }^{* *} \mathrm{P}<0.01$ (compared with B16-neo cells).

$(\mathrm{P}<0.01)$. We further performed a Matrigel invasion assay to qualitatively observe the effect of MUC1 expression on the invasive potential of cells. The results showed that the number of B16-MUC1 cells that invaded through the Matrigel-coated membrane was also significantly less than the number of invading B16-neo or B16 cells (Fig. 3C and D) (P<0.01). These results show that MUC1 expression inhibited cell migration and invasion in vitro.

MUC1-CT interacts with $\beta$-catenin and reduces the activity of $T$ cell factor $(T C F)$. Numerous reports have confirmed that MUC1 binds to $\beta$-catenin and is involved in the $\beta$-catenin signaling pathway. Therefore, we performed coimmunoprecipitation to investigate whether or not the interaction between MUC1 and $\beta$-catenin was also observed in B16-MUC1 cells. Immunoprecipitation of MUC1 from B16-MUC1 cell lysates using anti-MUC1-CT antibody (Ab-5) followed by immunoblot analysis using anti- $\beta$-catenin antibody revealed a protein band that co-migrated with $\beta$-catenin in total cell lysates; no $\beta$-catenin band was detected in the control immunoprecipitates with IgG (Fig. 4A). The results showed that MUC1-CT binds directly to $\beta$-catenin in B16-MUC1 cells. Since the Wnt pathway is known to be involved in tumor cell proliferation, to determine the effect of the interaction between the MUC1-CT and $\beta$-catenin on the activation of Wnt signaling, a luciferase reporter assay was performed. The results showed that Topflash/Fopflash reporter activity in B16-MUC1 cells was lower than that in the B16-neo cells $(\mathrm{P}<0.05)$ (Fig. 4B).
The result indicates that the interaction between MUC1-CT and $\beta$-catenin reduces the activity of TCF in B16-MUC1 cells when compared with that in the B16-neo cells.

MUC1 expression blocks $\beta$-catenin translocation to the nucleus. Since $\beta$-catenin is involved in MUC1 signal transduction, to evaluate the effect of MUC1 expression on $\beta$-catenin subcellular localization, equivalent protein aliquots of total cell lysates or purified nuclear or cytosolic fractions from cells were immunoblotted with the $\beta$-catenin antibody. Immunoblot analysis demonstrated that the total $\beta$-catenin levels were unchanged (Fig. 5A), the cytoplasmic $\beta$-catenin levels were increased (Fig. 5B) $(\mathrm{P}<0.05)$ and the nuclear $\beta$-catenin levels were reduced (Fig. 5C) $(\mathrm{P}<0.05)$ in $\mathrm{B} 16-\mathrm{MUC1}$ cells compared to B16-neo or B16 cells. The results indicate that MUC1 expression blocks the translocation of $\beta$-catenin to the nucleus. We also analyzed the level of E-cadherin, a molecular chaperone of $\beta$-catenin that plays an important role in cell adhesion. The immunoblot results show that E-cadherin expression was slightly upregulated in the MUC1-transfected B16 cells when compared with that of the negative control cells, although there was no statistical significance (Fig. 5D).

MUCl expression downregulates both cyclin DI and c-Myc. Nuclear translocation of $\beta$-catenin can activate cyclin D1 and c-Myc expression and stimulate cell proliferation. Our results demonstrated that MUC1 expression reduced levels of nuclear $\beta$-catenin and inhibited cell proliferation. Therefore, we carried 
A

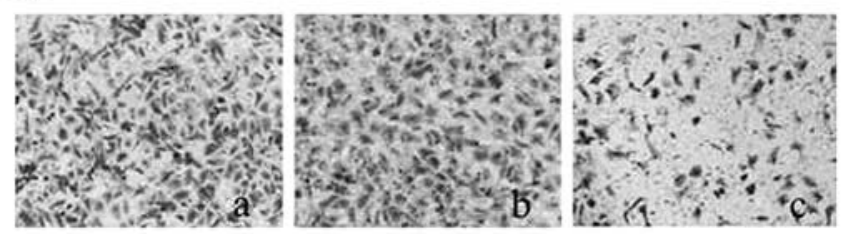

B

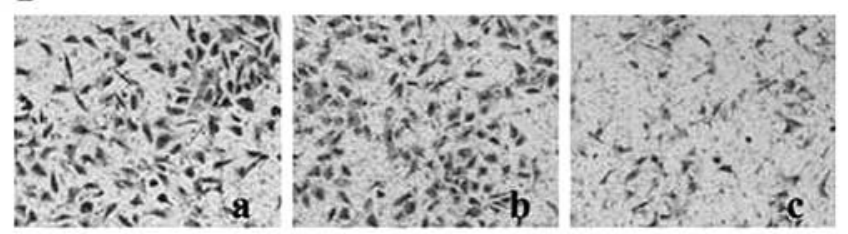

C
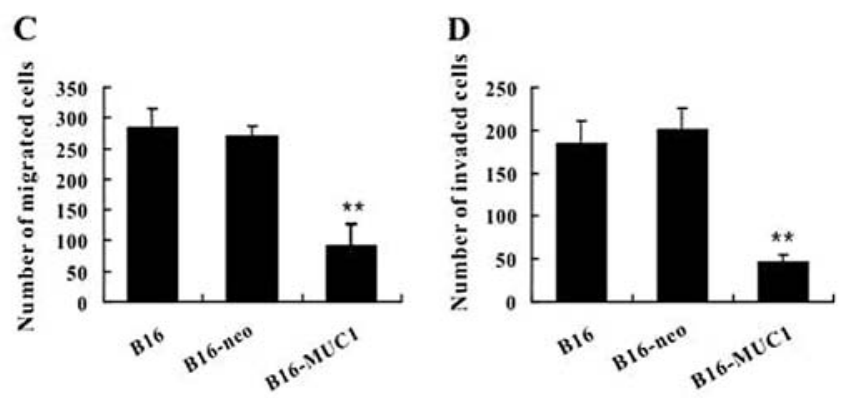

Figure 3. Mucin 1 (MUC1) expression inhibits cell migration and invasion Cell migration and Matrigel invasion assays were performed using Transwell chambers with $8-\mu \mathrm{m}$ pore size filters coated with or without Matrigel matrix in 24-well plates. Cells that migrated or invaded were counted in five random fields of each filter under a microscope. (A) Representative images of migrated cells detected by the Transwell migration assay. (B) Representative images of invaded cells detected by the Matrigel invasion assay: (a) B16 (b) B16-neo (c) B16-MUC1 9-23 cells. (C and D) The histograms represent the number of cells that migrated across the Transwell membranes or invaded through the Matrigel-coated membranes per field. Data represent the mean values of migrated or invaded cells of five random fields from three independent experiments. Statistically significant differences between B16-MUC1 9-23 and $\mathrm{B} 16$-neo cells are indicated $\left({ }^{* *} \mathrm{P}<0.01\right)$.

A

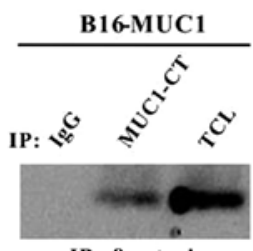

IB: $\beta$-catenin

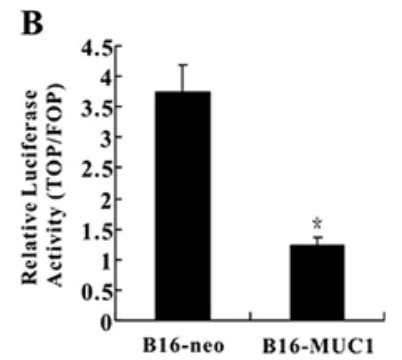

Figure 4. Mucin 1 (MUC1) interaction with $\beta$-catenin reduces the activity of T cell factor (TCF). (A) B16-MUC1 cell lysate was subjected to immunoprecipitation (IP) with the anti-MUC1-CT antibody or normal IgG and then immunoblotted (IB) with the anti- $\beta$-catenin antibody. TCL (Total cell lysate) was not subjected to immunoprecipitation. (B) B16-neo and B16-MUC1 cells were transiently transfected with TOPflash and FOPflash plasmids. The relative luciferase activity was calculated by the ratio of TOPflash/FOPflash luciferase activity, and each value was normalized to the luciferase activity of the internal control pRL-TK reporter plasmid. The results are presented as means \pm SD for 3 independent experiments.

out RT-PCR and western blotting to detect the expression of cyclin D1 and c-Myc. The PCR results showed that mRNA
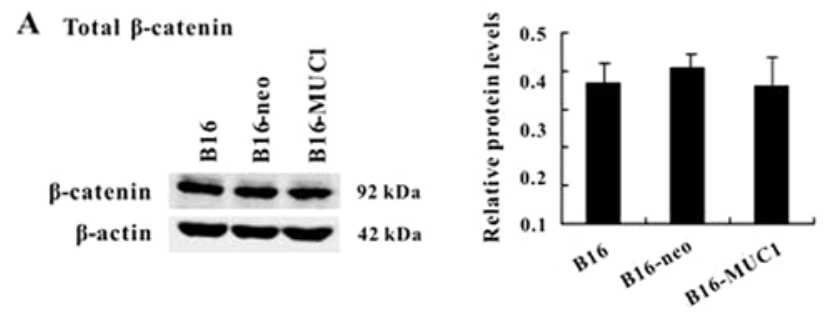

B
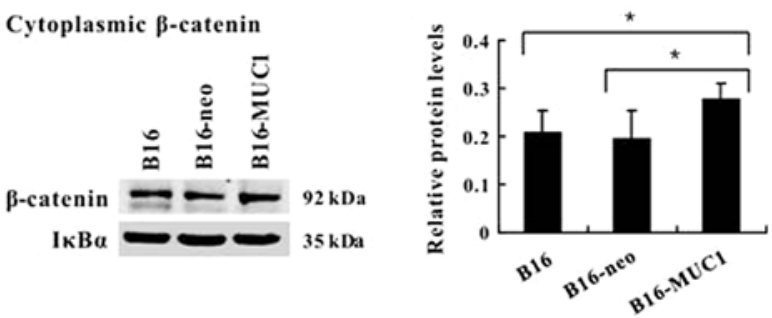

C Nuclear $\beta$-catenin
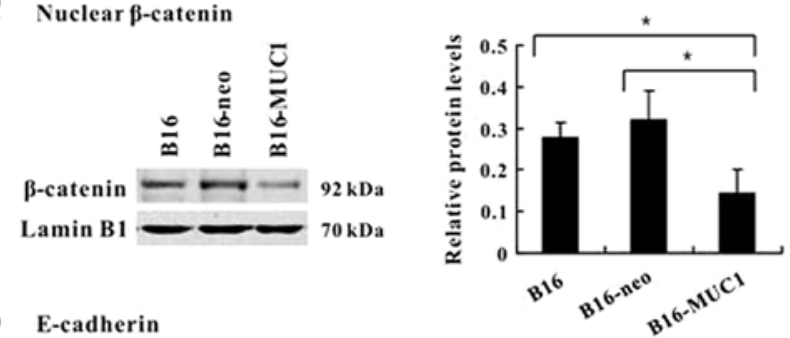

D E-cadherin

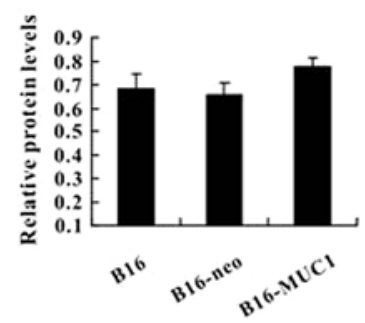

Figure 5. Mucin 1 (MUC1) expression increases cytoplasmic $\beta$-catenin levels and decreases nuclear translocation in transfected cells. (A) Immunoblotting of total $\beta$-catenin in total cell lysates. $\beta$-actin served as the internal control. (B) Cytoplasmic extracts were analyzed by western blotting for the expression of $\beta$-catenin. Cytoplasmic I $\mathrm{KB} \alpha$ was used as a protein loading control. (C) Nuclear extracts were detected by western blotting to assess the levels of nuclear $\beta$-catenin. Lamin B1 served as the nuclear loading control. (D) E-cadherin expression was detected by western blotting and normalized to $\beta$-actin. The bar charts represent relative protein levels calculated from the relative intensity ratio of total $\beta$-catenin/ $\beta$-actin, cytoplasmic $\beta$-catenin/IкB $\alpha$, nuclear $\beta$-catenin/Lamin B1 or cytoplasmic E-cadherin/ $\beta$-actin. Data are expressed as the means \pm SD of 3 independent experiments. ${ }^{*} \mathrm{P}<0.05$.

levels of cyclin D1 and c-Myc were significantly decreased in the B16-MUC1 cells when compared with levels in the B16 or B16-neo cells ( $\mathrm{P}<0.01$ and $\mathrm{P}<0.05$, respectively; Fig. 6A). Immunoblot analysis showed similar results (Fig. 6B). These findings indicate that MUC1 expression downregulated the levels of cyclin D1 and c-Myc.

MUC1 expression suppresses tumor growth in vivo. To evaluate the effects of MUC1 expression on tumorigenesis in vivo, B16-MUC1 and B16-neo cells $\left(2 \times 10^{6}\right)$ were inoculated subcutaneously into BALB/c nude mice to establish a subcutaneous transplant tumor model. Tumor growth was monitored for 12 days, and the B16-MUC1 tumors grew more slowly than the B16-neo tumors. The B16-MUC1 tumors were significantly 

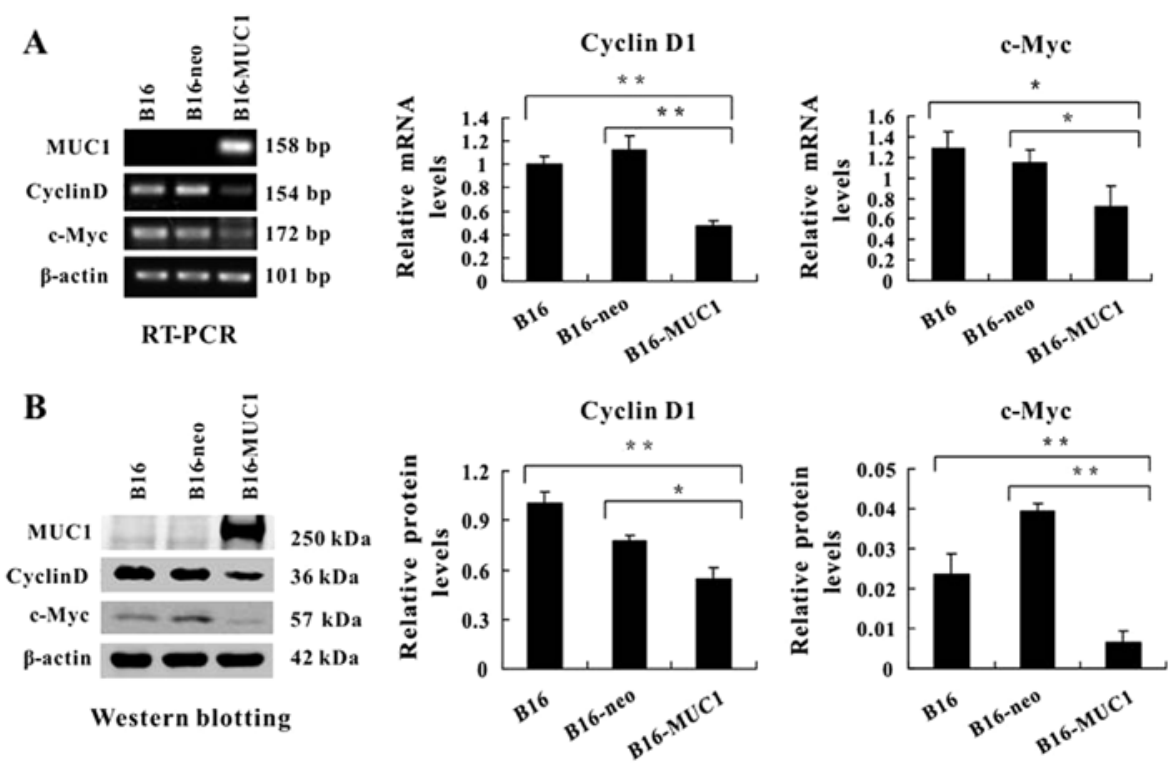

Figure 6. Mucin 1 (MUC1) expression downregulates both cyclin D1 and c-Myc. (A) mRNA levels of MUC1, cyclin D1 and c-Myc were detected by reverse transcription-polymerase chain reaction (RT-PCR) and normalized to $\beta$-actin. (B) Cell lysates were analyzed by western blotting for the expression of MUC1, cyclin D1 and c-Myc. $\beta$-actin was used as a loading control. Bar charts represent the relative mRNA and protein levels calculated from the relative intensity ratio of cyclin D1/ $\beta$-actin and c-Myc/ $\beta$-actin. Data are expressed as the means \pm SD of 3 independent experiments. ${ }^{*} \mathrm{P}<0.05,{ }^{* *} \mathrm{P}<0.01$.

A

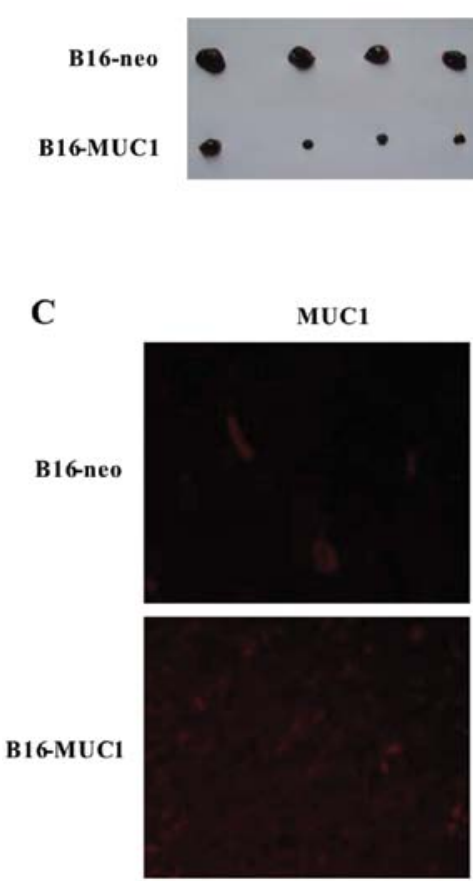

B

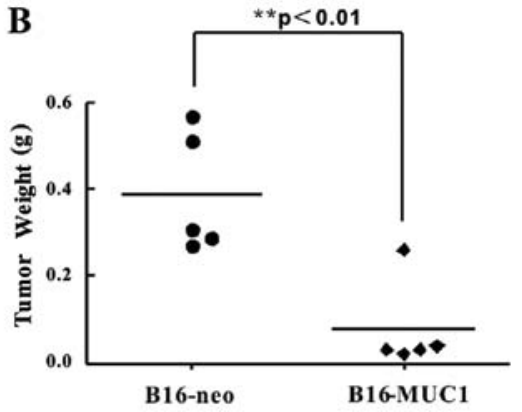

DAPI
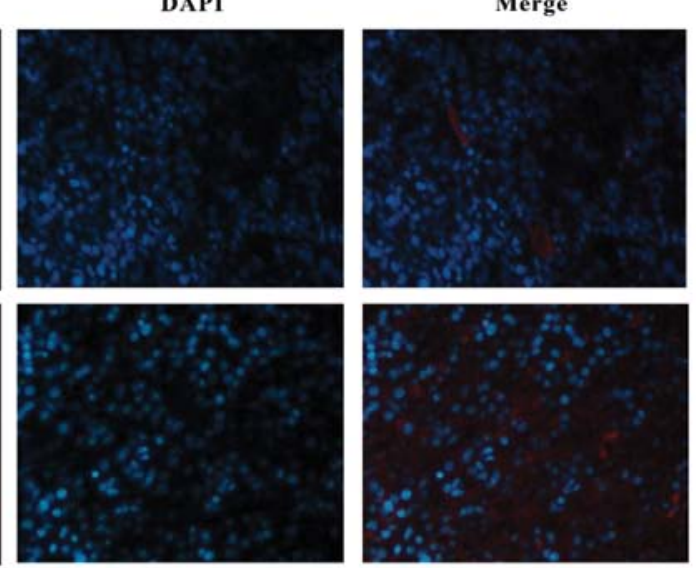

Figure 7. Mucin 1 (MUC1) expression inhibits tumor growth in BALB/c nude mice. B16-MUC1 and B16-neo cells (2x10 $\left.{ }^{6}\right)$ were injected into the right flank of $\mathrm{BALB} / \mathrm{c}$ nude mice to form subcutaneous tumors. On day 12 post injection, tumors were removed, weighed and photographed. (A) An image showing tumor sizes in the B16-MUC1 and B16-neo groups. (B) The scatter diagram represents the tumor weight as analyzed by GraphPad Prism 5 software. The lines represent the means $\pm \mathrm{SD} ;{ }^{* *} \mathrm{P}<0.01$. (C) MUC1 expression in tumor sections was detected by immunofluorescence staining with the anti-MUC1 primary antibody (GP1.4) and PE-conjugated secondary antibody (red). Nuclei were stained with DAPI (blue).

smaller than the B16-neo tumors, and the average weight of B16-MUC1 tumors $(0.08 \pm 0.05 \mathrm{~g})$ was significantly lower than that of the B16-neo tumors $(0.39 \pm 0.03 \mathrm{~g})$ (Fig. 7A and B)
$(\mathrm{P}<0.01)$. To determine whether MUC1 was expressed in the tumors, immunofluorescence staining was performed. The results showed strong positive staining for MUC1 in the 
B16-MUC1 tumors, while no MUC1 expression was detected in the B16-neo tumors (Fig. 7C). These results indicate that MUC1 expression in B16 cells significantly suppressed tumor growth in a BALB/c nude mouse transplant tumor model.

\section{Discussion}

In the present study, we investigated the effects of MUC1 on malignancy behavior both in vitro and in vivo by stable expression of human full-length MUC1 in the B16 mouse melanoma cell line. We established two MUC1-positive clones, B16-MUC1 9-12 and B16-MUC1 9-23, and one empty vector control clone, B16-neo. These cells were characterized in vitro for MUC1 expression, cell proliferation, cell cycle distribution, migration and invasion and evaluated in vivo for the effects of MUC1 expression on tumor growth in a mouse transplant tumor model.

We found that MUC1 expression in B16 cells significantly inhibited cell proliferation and induced cell cycle arrest. These results conflict with most previous reports showing that MUC1 is an oncogene (31-33). However, this is not the only report demonstrating that MUC1 expression is associated with inhibited cell proliferation; several published studies have shown similar results $(29,30)$. We also found that migration and invasion of B16-MUC1 cells were significantly decreased compared to B16 and B16-neo cells, opposing previous findings that MUC1 overexpression is associated with increased cell migration and invasion in breast, lung and pancreatic carcinoma cell lines $(34,35)$.

Several published studies have shown that the MUC1-CT can interact with $\beta$-catenin to form a complex that contributes to tumorigenesis and tumor progression $(36,37)$. Our present study showed that expression of the human full-length MUC1 in B16 cells increased the cytoplasmic levels of $\beta$-catenin, but reduced nuclear translocation of $\beta$-catenin and decreased cell proliferation. These results are similar to those described by Lillehoj et al (38), who showed that overexpression of MUC1 in HEK293T cells decreased the nuclear levels of $\beta$-catenin and inhibited cell proliferation. Cyclin D1 and c-Myc are two important transcriptional targets of the Wnt/ $\beta$-catenin pathway (39-41), both of which are involved in regulating cell cycle progression and promoting cellular proliferation and transformation. In our studies, MUC1 expression in B16 cells decreased the levels of nuclear $\beta$-catenin, reduced the activity of TCF, downregulated the expression of cyclin D1 and c-Myc and arrested the cell cycle at G1 phase. These results may provide a possible mechanistic explanation for how MUC1 expression decreased the proliferation of B16 cells in vitro. E-cadherin is a cell adhesion molecule that forms a complex with $\beta$-catenin and contributes to cell-cell adhesion (42), thereby preventing cell migration and invasion. Therefore, we examined the expression of E-cadherin in B16-MUC1, B16-neo and B16 cells. The results showed that E-cadherin expression was slightly increased in cells expressing MUC1 compared with the control cells, although the data did not reach statistical significance. These results suggest that the inhibition of cell migration and invasion may be associated with the upregulation of E-cadherin.

To investigate the effects of expressing human full-length MUC1 in B16 cells on tumorigenesis and tumor progression in vivo, a tumor growth assay was performed using BALB/c nude mice. We observed a strong reduction in the growth of B16-MUC1 tumors when compared with B16-neo tumors (Fig. 7A and B). These results agreed with the in vitro cell proliferation assays, suggesting that the decreased growth of MUC1-expressing primary tumors in nude mice is primarily due to decreased proliferative activity of the cells themselves. Premaratne et al (43) demonstrated that MUC1 expression in the prostate cancer cell line C4-2B4 had similar results. These findings suggest that MUC1 expression in the two types of cell lines displayed a negative effect on tumor growth, opposing most previous reports that MUC1 acts as an oncoprotein. Currently, there is no exact regulatory molecular mechanism to explain the conflicting data generated in different laboratories. Hattrup and Gendler (29) cautioned against overgeneralization of the results from individual cell lines on MUC1-mediated cancer progression since the functional regulation of $\mathrm{MUC1}$ in different cell lines may vary depending on diverse factors such as cell type and signaling context.

In addition, it is frequently assumed that the MUC1-CT functions as an oncogene, but the effect of the MUC1-N in cell transformation and tumorigenesis is not yet clear. Several reports show that the variable number tandem repeat (VNTR)-containing extracellular domain of MUC1 regulates the transcription of several genes (44), providing a new insight for understanding the function of MUC1-N. In a study conducted by Lillehoj et al (38), an engineered variant of the MUC1-CT, CD8/MUC1, that lacks the VNTR-containing extracellular domain was transfected into HEK293 cells resulting in decreased cell proliferation. In our study, we obtained similar results. Although we transfected full-length human MUC1 into B16 cells, it may merely be equivalent to transfection of the mouse MUC1-CT, since the homology with the human protein is only $34 \%$ in the extracellular tandem repeat domain, whereas it is $87 \%$ in the transmembrane and cytoplasmic domains (45). Moreover, the MUC1-CT is identical in normal and tumor cells. Based on these findings, we propose that the VNTR-containing extracellular domain of MUC1 may play an important role in regulating the tumorpromoting effects in various types of cancers, but further studies are needed.

In summary, we demonstrated that MUC1 expression in B16 cells inhibited cell proliferation, migration and invasion and suppressed tumor growth in a mouse transplant tumor model. These results may be associated with several MUC1related molecules of the $\beta$-catenin signaling pathway. It is suggested that the regulatory mechanisms of MUC1 as a oncoprotein are more complex than previously appreciated, which reinforces the importance of understanding alternative mechanisms that may regulate $\mathrm{MUC1}$.

\section{Acknowledgements}

We would like to thank Dr O.J. Finn for the pcDNA3-MUC1 plasmid, which was used to transfect the B16 cell line. This study was supported by grants from the China National Natural Science Foundation (no. 30972782) and the Major Development Programs for New Drugs of the Chinese Academy of Sciences during the 12th Five-Year Plan Period (no. 2011ZX09102-001-36). 


\section{References}

1. Kufe DW: Mucins in cancer: function, prognosis and therapy. Nat Rev Cancer 9: 874-885, 2009.

2. Gendler S, Taylor-Papadimitriou J, Duhig T, Rothbard J and Burchel J: A highly immunogenic region of a human polymorphic epithelial mucin expressed by carcinomas is made up of tandem repeats. J Biol Chem 263: 12820-12823, 1988

3. Ligtenberg MJ, Kruijshaar L, Buijs F, van Meijer M, Litvinov SV and Hilkens J: Cell-associated episialin is a complex containing two proteins derived from a common precursor. J Biol Chem 267 : 6171-6177, 1992.

4. Kufe DW: Targeting the human MUC1 oncoprotein: a tale of two proteins. Cancer Biol Ther 7: 81-84, 2008.

5. Li Y, Bharti A, Chen D, Gong J and Kufe D: Interaction of glycogen synthase kinase 3beta with the DF3/MUC1 carcinoma-associated antigen and beta-catenin. Mol Cell Biol 18: 7216-7224, 1998.

6. Li Y, Kuwahara H, Ren J, Wen G and Kufe D: The c-Src tyrosine kinase regulates signaling of the human DF3/MUC1 carcinomaassociated antigen with GSK3 beta and beta-catenin. J Biol Chem 276: 6061-6064, 2001.

7. Ren J, Li Y and Kufe D: Protein kinase C delta regulates function of the DF3/MUC1 carcinoma antigen in beta-catenin signaling. J Biol Chem 277: 17616-17622, 2002.

8. Raina D, Ahmad R, Kumar S, et al: MUC1 oncoprotein blocks nuclear targeting of $\mathrm{c}-\mathrm{Abl}$ in the apoptotic response to DNA damage. EMBO J 25: 3774-3783, 2006.

9. Wei X, Xu H and Kufe D: Human MUC1 oncoprotein regulates p53-responsive gene transcription in the genotoxic stress response. Cancer Cell 7: 167-178, 2005.

10. Wei X, Xu H and Kufe D: MUC1 oncoprotein stabilizes and activates estrogen receptor alpha. Mol Cell 21: 295-305, 2006.

11. Wei X, Xu H and Kufe D: Human mucin 1 oncoprotein represses transcription of the p53 tumor suppressor gene. Cancer Res 67: 1853-1858, 2007.

12. Yamamoto M, Bharti A, Li Y and Kufe D: Interaction of the DF3/MUC1 breast carcinoma-associated antigen and betacatenin in cell adhesion. J Biol Chem 272: 12492-12494, 1997.

13. Huang L, Chen D, Liu D, Yin L, Kharbanda S and Kufe D: MUC1 oncoprotein blocks glycogen synthase kinase 3beta-mediated phosphorylation and degradation of beta-catenin. Cancer Res 65: 10413-10422, 2005.

14. Ahmad R, Raina D and Trivedi V et al: MUC1 oncoprotein activates the IkappaB kinase beta complex and constitutive NF-kappaB signaling. Nat Cell Biol 9: 1419-1427, 2007.

15. Hu XF, Yang E, Li J and Xing PX: MUC1 cytoplasmic tail: a potential therapeutic target for ovarian carcinoma. Expert Rev Anticancer Ther 6: 1261-1271, 2006.

16. Kufe DW: Functional targeting of the MUC1 oncogene in human cancers. Cancer Biol Ther 8: 1197-1203, 2009.

17. Shin CY, Park KH, Ryu BK, Choi EY, Kim KC and Ko KH: Squamous differentiation downregulates Mucl mucin in hamster tracheal surface epithelial cell. Biochem. Biophys Res Commun 271: 641-646, 2000

18. Ligtenberg MJ, Buijs F, Vos HL and Hilkens J: Suppression of cellular aggregation by high levels of episialin. Cancer Res 52 : 2318-2324, 1992.

19. Wesseling J, van der Valk SW, Vos HL, Sonnenberg A and Hilkens J: Episialin (MUC1) overexpression inhibits integrinmediated cell adhesion to extracellular matrix components. J Cell Biol 129: 255-265, 1995.

20. Satoh S, Hinoda Y, Hayashi T, Burdick MD, Imai K and Hollingsworth MA: Enhancement of metastatic properties of pancreatic cancer cells by MUC1 gene encoding an anti-adhesion molecule. Int J Cancer 88: 507-518, 2000.

21. Regimbald LH, Pilarski LM, Longenecker BM, Reddish MA, Zimmermann G and Hugh JC: The breast mucin MUC1 as a novel adhesion ligand for endothelial intracellular adhesion molecule 1 in breast cancer. Cancer Res 56: 4244-4249, 1996.

22. Agrawal B and Longenecker BM: MUC1 mucin-mediated regulation of human T cells. Int Immunol 17: 391-399, 2005.

23. Li Y, Liu D, Chen D, Kharbanda S and Kufe D: Human DF3/MUC1 carcinoma-associated protein functions as an oncogene. Oncogene 22: 6107-6110, 2003.

24. Huang L, Ren J, Chen D, Li Y, Kharbanda S and Kufe D: MUC1 cytoplasmic domain coactivates Wnt target gene transcription and confers transformation. Cancer Biol Ther 2: 702-706, 2003 .
25. Raina D, Ahmad R, Chen D, Kumar S, Kharbanda S and Kufe D: MUC1 oncoprotein suppresses activation of the ARF-MDM2-p53 pathway. Cancer Biol Ther 7: 1959-1967, 2008

26. Li Y, Ahmad R, Kosugi M, et al: Survival of human multiple myeloma cells is dependent on MUC1 C-terminal transmembrane subunit oncoprotein function. Mol Pharmacol 78: 166-174, 2010.

27. Raina D, Kosugi M, Ahmad R, et al: Dependence on the MUC1-C oncoprotein in non-small cell lung cancer cells. Mol Cancer Ther 10: 806-816, 2011

28. Schroeder JA, Adriance MC, Thompson MC, Camenisch TD and Gendler SJ: MUC1 alters beta-catenin-dependent tumor formation and promotes cellular invasion. Oncogene 22: 1324-1332, 2003

29. Hattrup CL and Gendler SJ: MUC1 alters oncogenic events and transcription in human breast cancer cells. Breast Cancer Res 8: R37, 2006.

30. Costa NR, Paulo P, Caffrey T, Hollingsworth MA and SantosSilva F: Impact of MUC1 mucin downregulation in the phenotypic characteristics of MKN45 gastric carcinoma cell line. PLoS One 6: e26970, 2011.

31. Yuan Z, Liu X, Wong S, Machan JT and Chung MA: MUC1 knockdown with RNA interference inhibits pancreatic cancer growth. J Surg Res 157: e39-e46, 2009.

32. Schroeder JA, Masri AA, Adriance MC, et al: MUC1 overexpression results in mammary gland tumorigenesis and prolonged alveolar differentiation. Oncogene 23: 5739-5747, 2004.

33. Brossart P, Schneider A, Dill P, et al: The epithelial tumor antigen MUC1 is expressed in hematological malignancies and is recognized by MUC1-specific cytotoxic T-lymphocytes. Cancer Res 61: 6846-6850, 2001

34. Yuan Z, Wong S, Borrelli A and Chung MA: Down-regulation of MUC1 in cancer cells inhibits cell migration by promoting E-cadherin/catenin complex formation. Biochem Biophys Res Commun 362: 740-746, 2007.

35. Gao J, McConnell MJ, Yu B, et al: MUC1 is a downstream target of STAT3 and regulates lung cancer cell survival and invasion. Int J Oncol 35: 337-345, 2009.

36. Kufe D: Oncogenic function of the MUC1 receptor subunit in gene regulation. Oncogene 29: 5663-5666, 2010.

37. Retterspitz MF, Mönig SP, Schreckenberg S, et al: Expression of $\{$ beta\}-catenin, MUC1 and c-met in diffuse-type gastric carcinomas: correlations with tumour progression and prognosis. Anticancer Res 30: 4635-4641, 2010

38. Lillehoj EP, Lu W, Kiser T, Goldblum SE and Kim KC: MUC1 inhibits cell proliferation by a beta-catenin-dependent mechanism. Biochim Biophys Acta 1773: 1028-1038, 2007.

39. Tetsu $\mathrm{O}$ and McCormick F: Beta-catenin regulates expression of cyclin D1 in colon carcinoma cells. Nature 398: 422-426, 1999.

40. Sutherland RL and Musgrove EA: Cyclin D1 and mammary carcinoma: new insights from transgenic mouse models. Breast Cancer Res 4: 14-17, 2002.

41. Lavergne E, Hendaoui I, Coulouarn C, et al: Blocking Wnt signaling by SFRP-like molecules inhibits in vivo cell proliferation and tumor growth in cells carrying active $\beta$-catenin. Oncogene 30: 423-433, 2011.

42. Wijnhoven BP, Dinjens WN and Pignatelli M: E-cadherincatenin cell-cell adhesion complex and human cancer. Br J Surg 87: 992-1005, 2000.

43. Premaratne P, Welén K, Damber JE, Hansson GC and Bäckström M: $O$-glycosylation of MUC1 mucin in prostate cancer and the effects of its expression on tumor growth in a prostate cancer xenograft model. Tumour Biol 32: 203-213, 2011.

44. Cascio S, Zhang L and Finn OJ: MUC1 protein expression in tumor cells regulates transcription of proinflammatory cytokines by forming a complex with nuclear factor- $\mathrm{\kappa} \mathrm{B}$ p 65 and binding to cytokine promoters: importance of extracellular domain. J Biol Chem 286: 42248-42256, 2011

45. Spicer AP, Parry G, Patton S, and Gendler SJ: Molecular cloning and analysis of the mouse homologue of the tumor-associate mucin, MUC1, reveals conservation of potential $O$-glycosylation sites, transmembrane, and cytoplasmic domains and a loss of minisatellite-like polymorphism. J Biol Chem 266: 15099-15109, 1991. 